\title{
miR-495-3p Depresses Cell Proliferation and Migration By Down-Regulating HMGB1 in Colorectal Cancer
}

\section{Zhang Jieling ( $\nabla$ zjl863106131@163.com )}

wannan yi xue yuan di yi fu shu yi yuan: Yijishan Hospital of Wannan Medical College

Li Kai

Wannan Medical College

\section{Zheng Huifen}

wannan yi xue yuan di yi fu shu yi yuan: Yijishan Hospital of Wannan Medical College

\section{Zhu Yiping}

wannan yi xue yuan di yi fu shu yi yuan: Yijishan Hospital of Wannan Medical College

\section{Research Article}

Keywords: miR-495-3p, HMGB1, colorectal cancer, proliferation, migration

Posted Date: July 7th, 2021

DOI: https://doi.org/10.21203/rs.3.rs-663678/v1

License: (a) (i) This work is licensed under a Creative Commons Attribution 4.0 International License.

Read Full License 


\section{Abstract}

Background: MicroRNAs play an important role in the genesis and progression of tumors, including colorectal cancer (CRC), which has a high morbidity and mortality rate. In this research, the role of miR495-3p and HMGB1 in CRC was investigated.

Methods: We performed qRT-PCR to detect the expression of miR-495-3p in colorectal cancer tissues and cell lines. Functional experiments such as CCK-8 assay, EDU assay, Transwell assay and apoptosis assay were conducted to explore the effects of miR-495-3p on the proliferation, migration and apoptosis of CRC cells in vitro. Then, the use of database prediction, dual-luciferase reporter gene assay and functional experiments verified the role of miR-495-3p target gene HMGB1 in CRC. Finally, rescue experiments was performed to investigate whether overexpression of HMGB1 could reverse the inhibitory effect of miR495-3p on CRC cell proliferation in vivo and in vitro.

Results: miR-495-3p was down-regulated in colorectal cancer tissues and cell lines, and could inhibit the proliferation and migration of colorectal cancer cells, and promote cell apoptosis. The database prediction and dual-luciferase reporter gene assay showed that HMGB1 was the downstream target gene of miR-495-3p. We finally demonstrated that miR-495-3p inhibited CRC cell proliferation by targeting HMGB1 in vitro and in vivo.

Conclusion: Our research shows that miR-495-3p inhibits the progression of colorectal cancer by downregulating the expression of HMGB1, which indicates that miR-495-3p may become a potential therapeutic target for colorectal cancer.

\section{Introduction}

Colorectal cancer (CRC) is the third most common malignant tumor in the world, and second in cancerrelated mortality. In 2018, there were 1,096,601 new cases and 551,269 deaths [1]. The incidence of CRC in China is showing a younger trend [2]. The decline in the quality of life of patients and the huge medical expenses pose a threat to human health and social development. Although the diagnosis and treatment of CRC has been greatly improved in recent years, the five-year survival rate of patients has not been significantly improved [3], so it is urgent to improve the understanding of the exact mechanism of the disease and develop new targeted therapies.

MicroRNAs (miRNAs) are short non-coding RNAs with a length of approximately 22 nucleotides. At present, nearly 3,000 such molecules have been discovered and are still being explored. miRNAs participate in the regulation of post-transcriptional gene expression by binding to the 3'-UTR region of target mRNAs, leading to mRNA degradation or preventing its translation. A miRNA can target and regulate numerous mRNAs, and a target mRNA can also be regulated by multiple miRNAs, which have a complex network of relationships and play an important role in almost all biological pathways and regulate the occurrence and development of many human diseases, including cancer [4]. In recent decades, people's research on miRNAs is in full swing, especially the research on miRNAs in the 
occurrence and development of tumors. Studies have shown that miRNAs are dysregulated in a variety of cancers, and dysregulated miRNAs play a role in tumor cell proliferation, apoptosis, invasion and drug resistance, and act as cancer-promoting genes or suppressor genes. Based on this, miRNAs have also made corresponding progress in serving as targets for cancer diagnosis, monitoring and treatment [5]. According to previous studies, some scholars summarized hundreds of miRNAs with high or low expression in lung cancer, gastric cancer, breast cancer, liver cancer, etc., as well as the relevant mechanisms and pathways that affect the malignant progression of tumors, and discussed the use of these miRNAs as the corresponding cancer diagnosis and treatment targets and prognostic indicators [69]. Assuredly, there are also many reports about miRNAs in CRC. For example, it has been reported that miR-4319 can inhibit the proliferation, migration and invasion of CRC cells and inhibit the cell cycle, acting as a tumor suppressor gene in CRC by targeting ABTB1 [10]. MiR-629-5p is highly expressed in CRC tissues and cell lines, promotes the proliferation and migration of CRC cells, reduces the proportion of cell apoptosis through targeting CXXC4 and thus promotes the malignant progression of CRC [11]. Those suggested that differentially expressed miRNAs could be potential therapeutic targets for CRC. Differentially expressed miR-495-3p has been reported in various types of tumors, such as esophageal cancer [12], osteosarcoma [13], melanoma [14], etc. JW et al reported that miR-495-3p plays a tumor suppressive role in the development of gastric cancer by regulating a variety of epigenetic modifications [15]. It has been reported that miR-495-3p is regulated by the upstream molecule NEAT1 in CRC and plays a bridge role in the promotion of IncRNA in the development of CRC [16]. However, there are few studies on the differential expression of miR-495-3p in CRC, and at the same time, whether miR-495-3p can regulate cell proliferation in $\mathrm{CRC}$ and the related mechanism of action are still unclear.

High Mobility Group Box-1 (HMGB1) is a multifunctional non-histone protein composed of 215 amino acids and mainly distributed in the nucleus, which plays a key role in nucleosome structure and homeostasis, gene transcription, DNA recombination and damage repair, and maintenance of chromosome stability and telomere homeostasis. Studies have shown that HMGB1 plays an important role in promoting or suppressing cancer in various tumorigenesis including CRC [17]. We learned from the database that miR-495-3p had a complementary sequence with the 3'UTR end of HMGB1 mRNA. Therefore, we hypothesized that there is a close relationship between HMGB1 and miR-495-3p, thus influencing the behavior of CRC cells.

In this study, we explored the role of miR-495-3p in CRC and its molecular mechanism, hoping to develop new targets for the diagnosis and treatment of CRC. We found that miR-495-3p was significantly underexpressed in CRC tissues and cell lines. MiR-495-3p inhibited the proliferation and migration of CRC cells and promoted the apoptosis of CRC cells by down-regulating the expression of HMGB1, suggesting that miR-495-3p could be a potential target for the treatment of CRC.

\section{Materials And Methods}

\section{Acquisition of carcinoma and paracancerous tissue}


The CRC tissues and their paracancerous tissues were collected from 22 patients with colorectal cancer who had not undergone chemoradiotherapy in Yijishan Hospital. The tumor tissue and the surrounding normal tissue within $0.5 \mathrm{~cm}$ were collected during the resection and stored in liquid nitrogen immediately, with the informed consent of the patient and the approval of the medical committee and the ethics committee of Yijishan Hospital.

\section{Cell culture}

Four human CRC cell lines HT29, SW480, SW620, HCT116 and human normal colon epithelial cell lines FHC were purchased from Shanghai Cell Bank, Chinese Academy of Sciences (Shanghai, China). Cell complete medium contained 10\% FBS (Gibco, Waltham, America) and RPMI-1640(Gibco, Carlsbad, America) or DMEM (Hyclone, Logan, America). Four colorectal cancer cell lines were cultured in complete medium of RPMI-1640 while normal colon epithelial cells FHC were cultured in DMEM. The environment of the cell incubator was kept at a constant temperature of $37^{\circ} \mathrm{C}$ with $5 \%$ carbon dioxide. The cells were given fluid exchange or passage for 1-2 days.

\section{RNA extraction and reverse transcription-quantitative polymerase chain reaction (RT-qPCR)}

RNA of tissues and cells was extracted using Trizol (Invitrogen, Carlsbad, America) as per the merchant's step-by-step instructions. Nanodrop 2000 Spectrophotometer (Thermo Fisher, Waltham, America) was used to measure RNA concentration, and then 2 microliters were taken for reverse transcription using a reverse transcription kit (Takara, Dalian, China) to obtain the corresponding cDNA. Real-time fluorescence quantitative PCR was detected using QPCR kit (Takara, Dalian, China). The sequence of primers used in the experiment is as follows $₫ 66$, CTCGCTTCGGCAGCACA-forward and AACGCTTCACGAATTTGCGT-

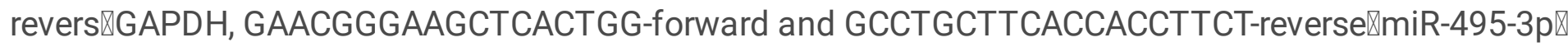
AAACAAACAUGGUGCACUUCUU-forward and GAAGUGCACCAUGUUUGUUUUU-reverse区HMGB1》 TGCTGATTAGTTACCACAGTTCTGA-forward and CTCGGGTACACAGGACACACAA- reverse. The primers

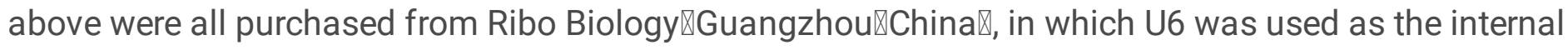
reference of miR-495-3p and GAPDH as the internal reference of HMGB1. The expression of miR-495-3p and HMGB1 in tissues or cells was analyzed by $2-\Delta \Delta \mathrm{CT}$ method.

\section{Cell transfection}

MiR-495-3p mimics, inhibitors and their respective negative controls (control mimic and control inhibitor), the short hairpin RNA(shRNA) HMGB1 and negative control (sh-NC) was synthesized by Ribo Biology (Guangzhou, China). The HMGB1 overexpression plasmid and the empty plasmid was supplied by GenePharma Co. Ltd (Shanghai, China). The original medium was replaced with the serum-free medium Opti-MEM (Gibco) when the confluence degree of cells in the six-well plate reached $60 \%-70 \%$. Then Lipofectamine 3000 Kit (Invitrogen) was used for transfection according to the manufacturer's instructions. After 6 hours, the Opti-MEM medium was replaced with medium containing $2 \%$ FBS or complete medium. 48 hours after transfection, cells were collected for subsequent experiments. 


\section{Cell Survival Test (CCK-8 assay)}

We used Cell Counting Kit-8 Kit (Keygen Biotech Co. Ltd,Nanjing, China) to analyze the viability of cells according to the instructions. After the corresponding transfection treatment, HT29 and SW480 cells were seeded into 96 -well plates with $1 \times 10^{4}$ cells per well. $10 \mu$ l of CCK-8 reagent was added to each well at 12 , $24,36,48$, and 60 hours after transfection. The cells were put back into the incubator for further incubation for 2 hours and the absorbance was measured at $450 \mathrm{~nm}$.

\section{Cell Proliferation Test (EDU assay)}

Six hours after transfection, the cells were inoculated into 24 -well plates. When the cells grew to $70 \%-80 \%$ concentration, EDU reagent was added to each well at a ratio of 1:1000 and incubated for two hours. Then the cells were fixed and stained according to the manufacturer's instructions (EDU Cell Proliferation Kit, Ribo, Guangzhou, China). Finally, fluorescence microscope was used to observe the cells and image them.

\section{Cell migration Test (Transwell assay)}

$24 \mathrm{~h}$ after transfection, the cells were digested, and the concentration of cells was adjusted to $1 \times 10^{5} \mathrm{cell} / \mathrm{s} / \mathrm{ml}$ with serum-free RPMI-1640. $100 \mu \mathrm{l}$ cell suspension were added to the upper compartment of Transwell chamber, while RPMI-1640 medium containing 20\% serum was added to the lower compartment. After incubation for 48 hours, the transmembrane cells were fixed with $4 \%$ paraformaldehyde and stained with $0.1 \%$ crystal violet, and the non-transmembrane cells were lightly wiped off with a cotton swab. Finally, an inverted microscope was used for observation and imaging.

\section{Cell apoptosis Test (flow cytometry)}

To detect apoptosis rates, we purchased apoptosis detection kits (BD Biosciences, CA, America). 48 hours after transfection, the cells were collected, washed with PBS for three times and resuspended with an appropriate amount of Binding Buffer. $1 \mu \mathrm{l}$ Annexin-V-Fluorescein isothiocyanate (FITC) was added for 15 min, and then $1 \mu$ Propidium iodide (PI) was added. (Each cell line was set with FITC staining alone, PI staining alone and blank control to adjust the drawing gate.) Finally, apoptosis was detected by flow cytometry.

\section{Correlation between miR-495-3p and HMGB1 (dual-luciferase reporter gene assay)}

To evaluate the direct binding of miR-495-3p to HMGB1, we used the dual luciferase reporter assay. After the wild-type and mutant-type vectors of HMGB1 3 '-UTR were designed, miR-495-3p mimic or control mimic and wild-type or mutant-type vector plasmid were co-transfected into 293T cells (it is a very common cell line expressing foreign genes for study, which is relatively easy to transfection). The cotransfection groups were as follows: miR-495-3p mimic + MUT, miR-495-3p mimic + WT, control mimic + 
MUT, and control mimic + WT. After 24 hours, a dual luciferase reporter assay kit was used for experimental analysis according to the instructions.

\section{In vivo experiment of nude mice (armpit tumor formation)}

We purchased 3-week-old SPF (special-solution-free) grade male nude mice from Hangzhou Ziyuan Laboratory Animal Science and Technology Co., Ltd. (Hangzhou, China). A one-week quarantine was conducted in the quarantine room of SPF animal laboratory of Wannan Medical College. About $2 \times 10^{6}$ HT29 cells were injected subcutaneously into the left axilla of each nude mouse. After that, the tumor volume was observed and measured every three days. After 15 days, the mice were sacrificed with cervical dislocation, and the tumor bodies were isolated and weighed. All operations have passed the experimental animal welfare and ethics review of Wannan Medical College.

\section{Protein extraction and Western blotting}

After 48 hours of transfection, the culture medium was sucked and discarded. $200 \mu$ of Radioimmunoreception (RIPA) lysis buffer (Thermo Fisher, Ma, America) containing protease inhibitor was added to each well of the six well plate, and then the protein in colorectal cancer cells was dissolved at $4{ }^{\circ} \mathrm{C}$ for 30 minutes. Then, the supernatant containing protein was centrifuged at $4^{\circ} \mathrm{C}$ under 7500 centrifugal force for $5 \mathrm{~min}$. Proteins were isolated by SDS-PAGE constant pressure 80-120V electrophoresis, and then transferred to PVDF membrane at constant current of $300 \mathrm{~mA}$. After sealed at room temperature for one hour, the required HMGB1 (24kDa) and internal reference actin (42kDa) protein bands were cut and placed into a 1:1000 ratio of primary antibody (ABclonal, Woburn, America) reaction solution for overnight incubation at $4{ }^{\circ} \mathrm{C}$. The next day, after washing the membrane with TBST for three times, the membrane was incubated in 1:5000 secondary antibody solution at room temperature for 2 hours. Exposure analysis was performed using a luminescent solution after washing the membrane again. Image $\mathrm{J}$ software is used to process the gray value of strips.

\section{Data analysis method}

According to the mean and standard deviation of the three independent repeated experiments, GraphPad Prism-8 software was used for analysis and mapping. The corresponding $\mathrm{P}$ value was calculated by $\mathrm{t}-$ test. $P<0.05$ indicated statistically significant difference, and was represented by $*, P<0.01$ was denoted by $\star \star, P<0.001$ is denoted by $* \star *$.

\section{Results}

\section{miR-495-3p expression is decreased in CRC tissues and cell lines.}

We used RT-qPCR to detect the expression level of miR-495-3p in 22 pairs of CRC tissues and their paracancer tissues, four types of CRC cells (HT29,SW480,SW620,HCT116) and one normal colon epithelial cell (FHC). The results showed that the expression of miR-495-3p was significantly lower in CRC 
tissues compared with the corresponding paracancerous tissues (Fig. 1A). Compared with normal intestinal epithelial cells, the level of miR-495-3p in CRC cells was also prominently reduced (Fig. 1B), and the differential expressions of HT29 and SW480 were the most significant. Therefore, these two cell lines were selected to complete the following experiment. In order to further understand the specific role of miR-495-3p in CRC, we transfected miR-495-3p mimic, control mimic, miR-495-3p inhibitor and control inhibitor into HT29 and SW480 respectively to complete the high and low expression of miR-495-3p. RTqPCR results showed that this step was obviously successful (Fig.1C). At this point, we learned that miR495-3p was obviously under-expressed in CRC tissues and cell lines.

\section{miR-495-3p inhibits the proliferation, migration and promote apoptosis of CRC cells}

In order to investigate the effect of miR-495-3p on the proliferation of colorectal cancer cells, we performed CCK-8 and EDU experiments on CRC cells (HT29 and SW480) transfected with miR-495-3p mimic and inhibitor and their respective control groups. The results showed that compared with the control group, the overexpression of miR-495-3p significantly reduced the proliferation ability of cells, while the proliferation ability of cells in the low-expression group of miR-495-3p was increased (Fig. 2AC). Meanwhile, the Transwell assay was used to explore the effect of miR-495-3p on the migration of CRC cells. As shown in the figures, fewer migrated cells were found in the group with increased miR-495-3p expression compared with the control group, while more migrated cells were found in the group with decreased miR-495-3p expression compared with the control group (Fig. 2D). The next experiment was to detect the influence of miR-495-3p on the apoptosis of CRC cells by flow cytometry. As we suspected, the proportion of cells apoptotic after overexpression of miR-495-3p was increased, while the proportion was decreased after knocking-down of miR-495-3p, compared with the respective control group (Fig. 2E). In general, we proved that miR-495-3p could inhibit the proliferation and migration of CRC cells and promote cell apoptosis, while knockdown of miR-495-3p could achieve the contrary result.

\section{HMGB1 is the target gene of miR-495-3p, with an abundant expression in CRC.}

Up to now, we have confirmed that miR-495-3p could inhibit the malignant biological behavior of CRC cells. In order to explore the mechanism of miR-495-3p's action more specifically, we used two databases (TargetScan and RNAhybrid) to predict the downstream target genes of miR-495-3p. We discovered that there were complementary sequences in the $3^{\prime} U T R$ of HMGB1 mRNA and miR-495-3p, which may be the direct binding site of the two molecule (Fig. 3A). To verify this hypothesis, we constructed HMGB1 wildtype and mutant-type luciferase reporter gene plasmids, and then co-transfected these plasmids into 293T cells with miR-495-3p mimic and control mimic. In HMGB1 3'UTR wild-type, luciferase activity was distinctly reduced after overexpression of miR-495-3p compared with the control group. However, in the mutants whose binding sequences were changed, there was no significant difference in luciferase activity between the over-expression group of miR-495-3p and the control group (Fig. 3B). After transfection with miR-495-3p overexpression,knockdown and corresponding controls, we detected the expression of HMGB1 protein by Western blotting and HMGB1 mRNA by qPCR. Results as we expected, compared with the control group, the protein and mRNA levels of HMGB1 decreased after overexpression of miR-495-3p, 
while the protein and mRNA levels of HMGB1 increased after decreased expression of miR-495-3p (Fig. 3C, D). Go a step further, HMGB1 is the target of miR-495-3p. After that, we found that the expression of HMGB1 was up-regulated in CRC by TCGA database (Fig.3E). Meanwhile, the level of HMGB1 in five CRC cell lines was detected by qPCR. Compared with FHC, the level of HMGB1 in four CRC cells was significantly increased. Among them, the differential expression of HT29 and SW480 was the most obvious, which was consistent with the cell lines with the most significant differential expression of miR495-3p (Fig.3F), suggesting that HMGB1 was highly expressed in CRC. On the whole, HMGB1, which is highly expressed in CRC, is the target gene of miR-495-3p. To further study the orientation that HMGB1 contributes to CRC progression, three sh-RNAs and the overexpressed plasmid of HMGB1 were constructed. Figure 3.1 shows the knockdown and overexpression efficiency of HMGB1. Among the three sh-HMGB1, the knockdown efficiency of sh-HMGB1-3 is the highest, so we chose sh-HMGB1-3 to complete the knockdown intervention step.

\section{HMGB1 promotes the proliferation and migration of CRC cells and inhibits apoptosis}

After HMGB1 knockdown and overexpression, cell proliferation was also detected by CCK-8 and EDU. Transwell assay was used to detect cell migration and cell apoptosis was detected by flow cytometry. CCK-8 and EDU assays revealed that, compared with the control group, the proliferation ability of cells in the HMGB1 knockdown group was decreased, while the proliferation ability was increased in the overexpression group (Fig.4A-C). Transwell results indicated that reduced HMGB1 expression resulted in reduced migratory cells, while higher HMGB1 expression resulted in a corresponding increase in migratory cells (Fig.4D). Subsequent flow cytometry manifested that knockdown of HMGB1 inhibited apoptosis, while overexpression of HMGB1 had the opposite affect (Fig. 4E). In short, HMGB1 promotes proliferation, migration and inhibits apoptosis of CRC cells.

\section{miR-495-3p inhibits proliferation and migration of CRC by targeting HMGB1 in vivo and in vitro.}

Rescue experiments were conducted to further verify the function of miR-495-3p by targeting HMGB1. Western blotting showed that the protein level of HMGB1 decreased significantly after overexpression of miR-495-3p alone, and increased correspondingly after overexpression of HMGB1 alone, while the protein level of HMGB1 was between the two when overexpression of miR-495-3p and HMGB1 at the same time, which indicated that co-transfection of miR-495-3p mimic and HMGB1 overexpression plasmid could weaken the negative regulation of miR-495-3p on HMGB1 (Fig.5A). Further functional experiments CCK-8 showed that overexpression of HMGB1 attenuated the inhibitory effect of miR-495-3p on the proliferation of CRC cells compared to negative controls (Fig.5B). Transwell experiments also confirmed that the inhibitory effect of miR-495-3p on the migration of CRC cells was weakened when HMGB1 and miR-495$3 p$ were overexpressed synchronously (Fig.5C,D). These results suggest that miR-495-3p can inhibit the proliferation and migration of CRC cells by targeting HMGB1 in vitro. In order to elucidate the impact and mechanism of miR-495-3p on tumor formation in vivo, HT29 cells stably transfected with miR-495-3p overexpression or / and HMGB1 overexpression were inoculated subcutaneously into the left armpit of nude mice (Fig. 5E). According to the experiment, compared with the control lentivirus group, tumor 
weight and volume in the miR-495-3p lentivirus group were significantly decreased, and tumor weight and volume in the HMGB1 lentivirus group were significantly increased. However, the tumor volume and weight of miR-495-3p\&HMGB1 lentivirus group were between the former two groups (Fig.5F-H). Results from Western blot of tumor tissues displayed the lowest expression of HMGB1 protein in the miR-495-3p lentivirus group and the highest in the HMGB1 lentivirus group. Similarly, HMGB1 protein level of the cooverexpression group of miR-495-3p and HMGB1 was located between miR-495-3p overexpressed alone and HMGB1 overexpressed alone (Fig. 5J). These results indicate that miR-495-3p can inhibit colorectal tumor growth in vivo while HMGB1 promotes tumor growth, and further indicate that miR-495-3p can inhibit CRC cell proliferation by targeting HMGB1.

\section{Discussion}

miR-495-3p is involved in many pathophysiological processes. A research verified that miR-495-3p can play a role in intervertebral disc degeneration (IVDD) through inhibiting inflammation and apoptosis of human nucleus pulposus cells by targeting IL5RA [18]. Another study also suggested that miR-495-3p may be related to lung function and chronic obstructive pulmonary emphysema (COPD) [19]. Definitely, miR-495-3p has been extensively studied in cancer, including the occurrence, development and drug resistance of cancer. A research clarified that functional loss or inhibition of miR-495-3p can trigger the overexpression of a variety of oncogenic epigenetic modifiers (EMS), thereby promoting malignant transformation and growth of gastric epithelial cells [15]. Chen et al. found that miR-495-3p inhibited multidrug resistance of gastric cancer by regulating autophagy through GRP78/mTOR axis [20]. As far as we know, few researchers have reported the specific role and mechanism of miR-495-3p in CRC. In this study, we found that the expression of miR-495-3p was decreased in CRC tissues and cells, and artificial intervention of miR-495-3p expression would affect the malignant phenotype of CRC cells. After the overexpression of miR-495-3p, the proliferation and migration ability of CRC cells decreased and the proportion of apoptosis increased. Conversely, miR-495-3p knockdown increased the proliferation and migration ability, and reduced the proportion of apoptosis. These results suggest that miR-495-3p may play a role as a tumor suppressor gene in CRC.

In order to further explore the potential mechanism of miR-495-3p in CRC, we concluded that HMGB1 might be the target of miR-495-3p through bioinformatics analysis. HMGB1 consists of 215 amino acids with two DNA binding domains (Box A and Box B) and an acidic tail. Among them, Box A played an antagonistic role, while Box B played A cytokine inducing role. HMGB1 binds to DNA through the DNA binding domain and regulates the structure of chromosomes, thereby regulating gene transcription [21]. Overexpression and abnormal secretion of HMGB1 play an important role in many diseases. Previous studies have shown that HMGB1 plays a role in sepsis, atherosclerosis, rheumatoid arthritis and other inflammation-related diseases [22]. In recent years, many studies have focused on its role in a variety of cancers, including CRC. Various reports have shown that HMGB1 expression is significantly up-regulated in breast cancer, gastric cancer, lung cancer and other cancers, and down-regulated in pancreatic cancer [23-26]. Ueda et al. examined the expression of HMGB1 in cancer tissues and normal paracancerous tissues of 140 patients with CRC using qPCR, and found that the expression of HMGB1 in tumor tissues 
was significantly higher than that in normal tissues, and the high expression of HMGB1 was closely associated with larger tumor volume, higher lymph node metastasis rate and lower survival rate [27]. In our research, we also confirmed the high expression of HMGB1 in CRC tissues and cells, and the high expression of HMGB1 can promote the proliferation and migration of CRC cells. We also revealed the negative regulatory relationship between HMGB1 and miR-495-3p, and confirmed that HMGB1 was the downstream target of miR-495-3p by dual-luciferase reporter gene assay. We also found that the expression of HMGB1 protein and mRNA were significantly decreased after the up-regulation of miR-495$3 p$. Inversely, HMGB1 protein and mRNA levels were significantly increased after down-regulation of miR495-3p. Further rescue experiments showed that, compared with the control group, HMGB1 could reduce the inhibitory effect of overexpression of miR-495-3p on the proliferation and migration of CRC cells, and this consequence was also verified in vivo by our tumor formation experiment in nude mice. Those suggests that miR-495-3p plays a role as a tumor suppressor gene in CRC by targeting HMGB1 in vivo and in vitro.

Broadly speaking, we confirmed the low expression of miR-495-3p in CRC and high expression of HMGB1 in CRC. Moreover, miR-495-3p regulates the proliferation, migration and apoptosis of CRC cells by targeting HMGB1 in vivo and in vitro, revealing the expectation that miR-495-3p could be used as a potential therapeutic target for CRC. In other words, we have discovered a new regulatory network that affects CRC, and the downstream pathways of this regulatory pathway may be studied in detail in the future.

\section{Declarations}

\section{Ethics approval and consent to participate}

The experimental animal program involved has been approved by the Experimental Animal Welfare and Ethics Committee of Wannan Medical College.

\section{Consent for publication}

Not applicable

\section{Availability of data and materials}

All authors ensure that our data does not contain any of the following:

(a) Infringes or makes unauthorized use of the Intellectual Property Rights or

any other right of any person;

(b) Is defamatory, derogatory, discriminatory, or violates any rights of privacy;

(c) Breaches any applicable law or regulation; 
(d) Contains a virus, malware, or other potentially harmful component, information, or instructions; (e) Is indecent, obscene, or offensive. Please confirm agreement with this statement.

\section{Competing interests}

The authors declare no competing interests.

\section{Funding}

The authors received no funding for this work.

\section{Authors' contributions}

Jieling Zhang completed most of the experiments, and Kai Li assisted in completing the experiments. Huifen Zheng completed data collection and statistics. Yiping Zhu designed and supervised the completion of this experiment and wrote this article. The authors read and approved the final manuscript.

\section{Acknowledgements}

Not applicable

\section{References}

1. Bray F, Ferlay J, Soerjomataram I, Siegel RL, Torre LA, Jemal A. Global cancer statistics 2018: GLOBOCAN estimates of incidence and mortality worldwide for 36 cancers in 185 countries. CA Cancer J Clin. 2018. 68(6): 394-424.

2. Siegel RL, Miller KD, Fedewa SA, et al. Colorectal cancer statistics, 2017. CA Cancer J Clin. 2017. 67(3): 177-193.

3. Dienstmann R, Salazar R, Tabernero J. Personalizing colon cancer adjuvant therapy: selecting optimal treatments for individual patients. J Clin Oncol. 2015. 33(16): 1787-96.

4. Acunzo M, Romano G, Wernicke D, Croce CM. MicroRNA and cancer-a brief overview. Adv Biol Regul. 2015 Jan; 57:1-9.

5. Iorio MV, Croce $\mathrm{CM}$. MicroRNA dysregulation in cancer: diagnostics, monitoring and therapeutics. A comprehensive review. EMBO Mol Med. 2012 Mar;4(3):143-59.

6. Iqbal MA, Arora S, Prakasam G, Calin GA, Syed MA. MicroRNA in lung cancer: role, mechanisms, pathways and therapeutic relevance. Mol Aspects Med. 2019 Dec;70:3-20.

7. Shin VY, Chu KM. MiRNA as potential biomarkers and therapeutic targets for gastric cancer. World J Gastroenterol. 2014 Aug 14;20(30):10432-9.

8. Kandettu A, Radhakrishnan R, Chakrabarty S, Sriharikrishnaa S, Kabekkodu SP. The emerging role of miRNA clusters in breast cancer progression. Biochim Biophys Acta Rev Cancer. 2020 Dec;1874(2):188413. 
9. Nagy Á, Lánczky A, Menyhárt O, Győrffy B. Validation of miRNA prognostic power in hepatocellular carcinoma using expression data of independent datasets. Sci Rep. 2018 Jun 15;8(1):9227.

10. Huang L, Zhang Y, Li Z, Zhao X, Xi Z, Chen H, Shi H, Xin T, Shen R, Wang T. MiR-4319 suppresses colorectal cancer progression by targeting ABTB1. United European Gastroenterol J. 2019 May;7(4):517-528.

11. Lu J, Lu S, Li J, Yu Q, Liu L, Li Q. MiR-629-5p promotes colorectal cancer progression through targetting CXXC finger protein 4. Biosci Rep. 2018 Aug 29;38(4):BSR20180613.

12. Huang GM, Zang HL, Geng YX, Li YH. LncRNA FAM83A-AS1 aggravates the malignant development of esophageal cancer by binding to miR-495-3p. Eur Rev Med Pharmacol Sci. 2020 Sep;24(18):94089415 .

13. Zhao G, Zhang L, Qian D, Sun Y, Liu W. miR-495-3p inhibits the cell proliferation, invasion and migration of osteosarcoma by targeting C1q/TNF-related protein 3. Onco Targets Ther. 2019 Aug 2;12:6133-6143.

14. Xia Y, Zhou Y, Han H, Li P, Wei W. IncRNA NEAT1 facilitates melanoma cell proliferation, migration, and invasion via regulating miR-495-3p and E2F3. J Cell Physiol. 2019;234(11):19592-19601.

15. Eun JW, Kim HS, Shen Q, Yang HD, Kim SY, Yoon JH, Park WS, Lee JY, Nam SW. MicroRNA-495-3p functions as a tumor suppressor by regulating multiple epigenetic modifiers in gastric carcinogenesis. J Pathol. 2018 Jan;244(1):107-119.

16. He Z, Dang J, Song A, Cui X, Ma Z, Zhang Z. NEAT1 promotes colon cancer progression through sponging miR-495-3p and activating CDK6 in vitro and in vivo. J Cell Physiol. 2019 Nov;234(11):19582-19591.

17. Kang R, Zhang Q, Zeh HJ 3rd, Lotze MT, Tang D. HMGB1 in cancer: good, bad, or both? Clin Cancer Res. 2013 Aug 1;19(15):4046-57.

18. Lin X, Lin Q. MiRNA-495-3p Attenuates TNF-a Induced Apoptosis and Inflammation in Human Nucleus Pulposus Cells by Targeting IL5RA. Inflammation. 2020 Oct;43(5):1797-1805.

19. Zhou T, Yu Q, Sun C, Wang Y, Zhong Y, Wang G. A pilot study of blood microRNAs and lung function in young healthy adults with fine particulate matter exposure. J Thorac Dis. 2018;10(12):7073-7080.

20. Chen S, Wu J, Jiao K, Wu Q, Ma J, Chen D, Kang J, Zhao G, Shi Y, Fan D, Zhao G. MicroRNA-495-3p inhibits multidrug resistance by modulating autophagy through GRP78/mTOR axis in gastric cancer. Cell Death Dis. 2018 Oct 19;9(11):1070.

21. Cheng KJ, Alshawsh MA, Mejia Mohamed EH, Thavagnanam S, Sinniah A, Ibrahim ZA. HMGB1: an overview of its versatile roles in the pathogenesis of colorectal cancer. Cell Oncol (Dordr). 2020 Apr;43(2):177-193.

22. H. Yang, H. Wang, S.S. Chavan, U. Andersson, High mobility group box protein 1 (HMGB1): The prototypical endogenous danger molecule. Mol Med 21(Suppl 1), S6-S12 (2015)

23. Wang XH, Zhang SY, Shi M, Xu XP. HMGB1 Promotes the Proliferation and Metastasis of Lung Cancer by Activating the Wnt/ $\beta$-Catenin Pathway. Technol Cancer Res Treat. 2020 JanDec;19:1533033820948054. 
24. Wang CQ, Huang BF, Wang Y, Hu GR, Wang Q, Shao JK. [Expression of HMGB1 protein in breast cancer and its clinicopathological significance]. Zhonghua Bing Li Xue Za Zhi. 2020 Jan 8;49(1):5761.

25. Fang J, Ge X, Xu W, Xie J, Qin Z, Shi L, Yin W, Bian M, Wang H. Bioinformatics analysis of the prognosis and biological significance of HMGB1, HMGB2, and HMGB3 in gastric cancer. J Cell Physiol. 2020 Apr;235(4):3438-3446.

26. Kang R, Xie Y, Zhang Q, Hou W, Jiang Q, Zhu S, Liu J, Zeng D, Wang H, Bartlett DL, Billiar TR, Zeh HJ 3rd, Lotze MT, Tang D. Intracellular HMGB1 as a novel tumor suppressor of pancreatic cancer. Cell Res. 2017 Jul;27(7):916-932.

27. Ueda M, Takahashi Y, Shinden Y, Sakimura S, Hirata H, Uchi R, Takano Y, Kurashige J, Iguchi T, Eguchi $\mathrm{H}$, Sugimachi K, Yamamoto H, Doki Y, Mori M, Mimori K. Prognostic significance of high mobility group box 1 (HMGB1) expression in patients with colorectal cancer. Anticancer Res. 2014 Oct;34(10):5357-62.

\section{Figures}

A

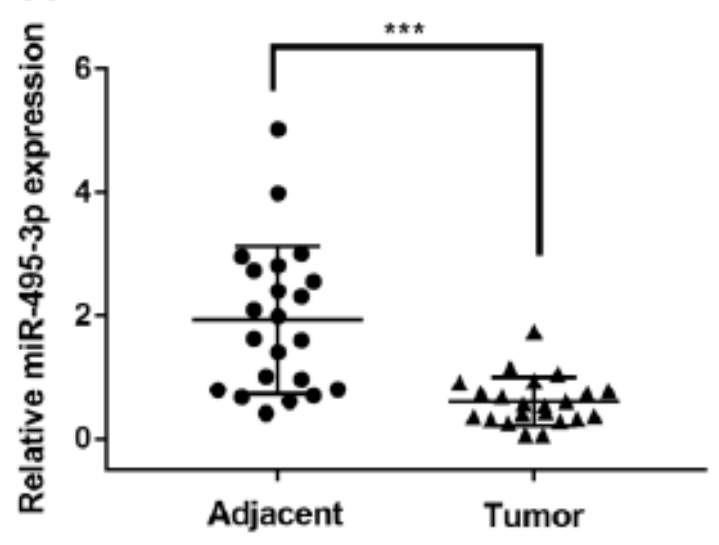

C HT29

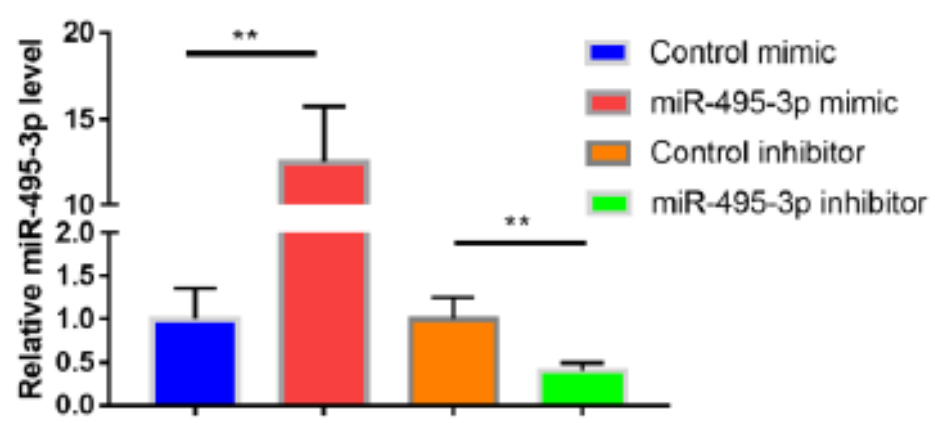

B

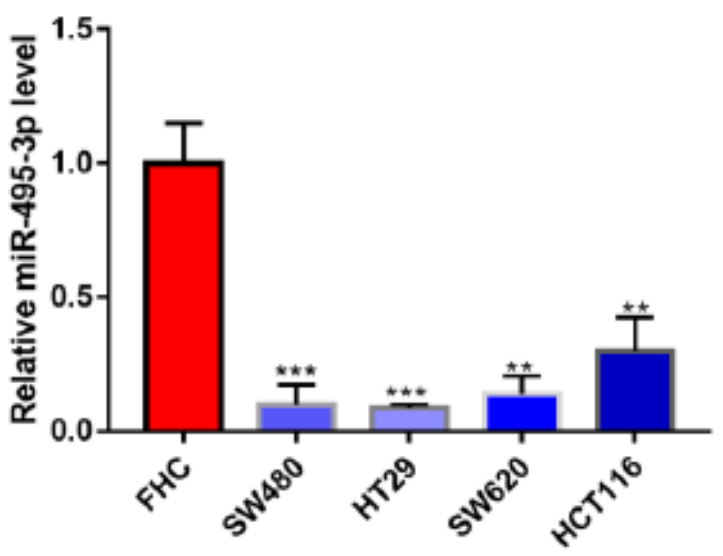

SW480

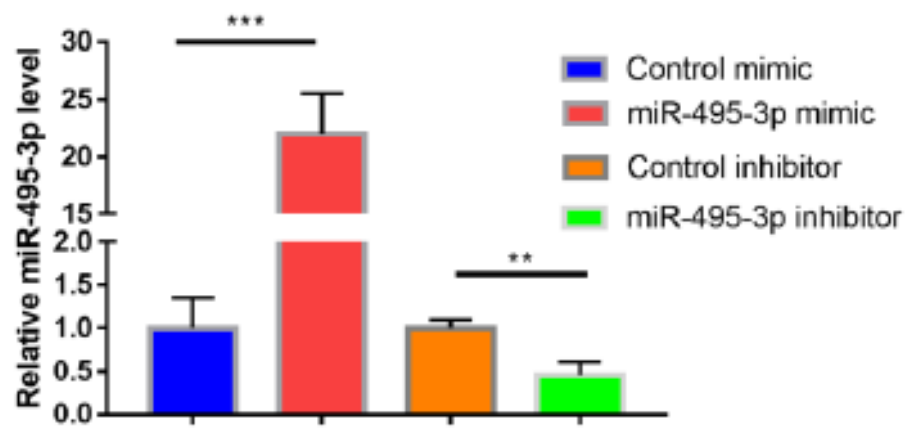

Figure 1 
miR-495-3p is underexpressed in colorectal cancer. (a) miR-495-3p levels in 22 pairs of CRC tissues and their paracancer tissues assessed by RT-qPCR. (b) miR-495-3p levels in HT29, SW480, SW620, HCT116 and FHC cells assessed by RT-qPCR. (c) miR-495-3p levels in HT29 and sw480 cells transfected with the control mimic, miR-495-3p mimic, control inhibitor, or miR-495-3p inhibitor assessed by RT-qPCR. RTqPCR, real-time quantitative polymerase chain reaction. ${ }^{*} p<0.05$, $* * p<0.01$, and $* * * p<0.001$
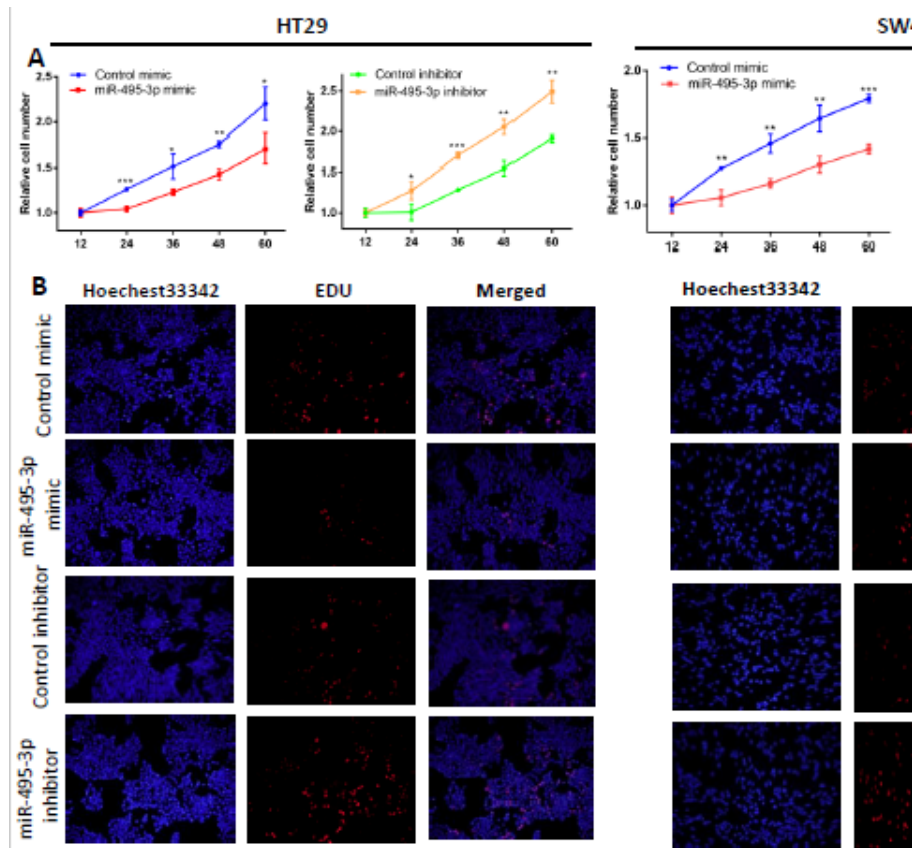

SW480
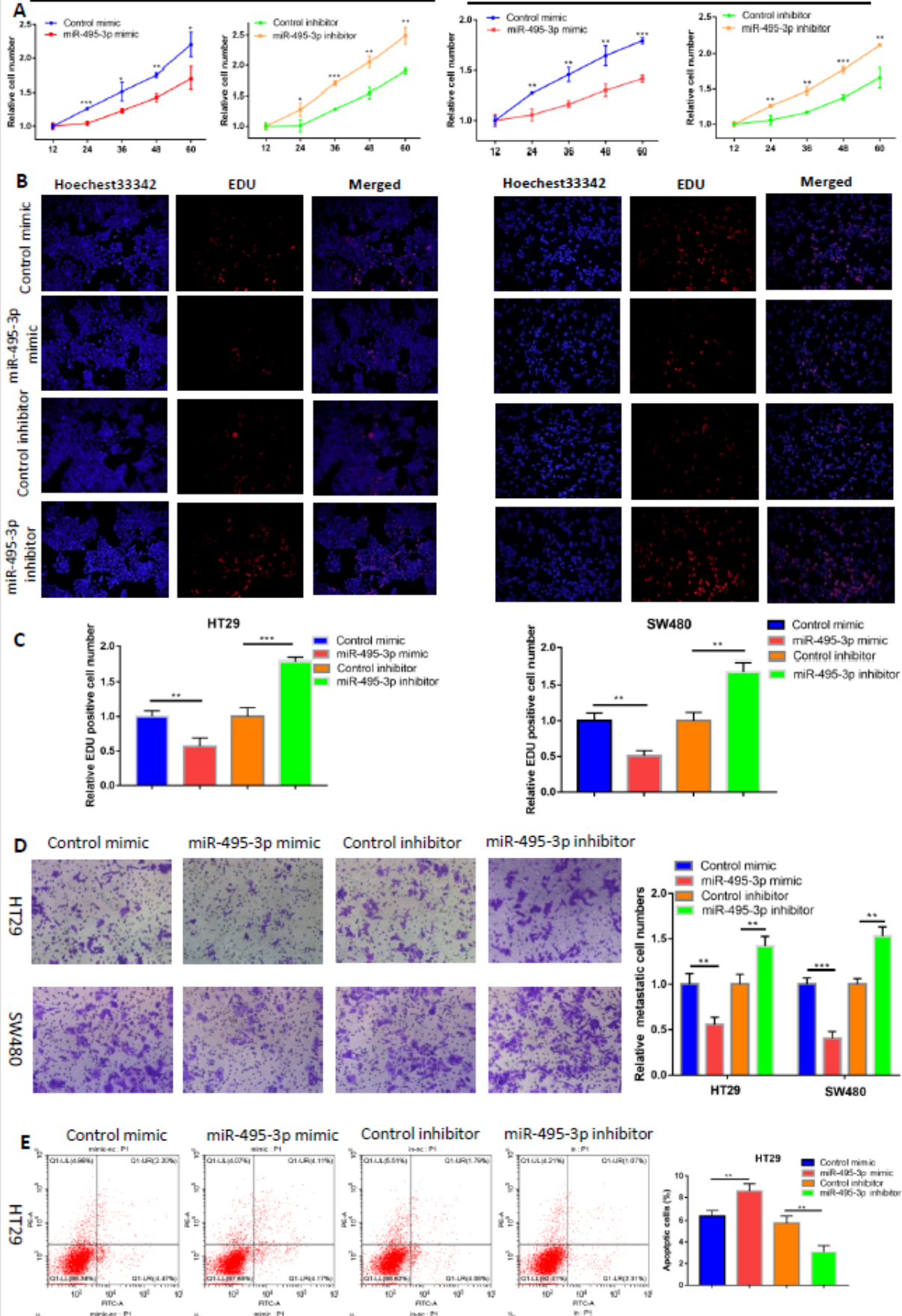

miR-495-3p inhibitor
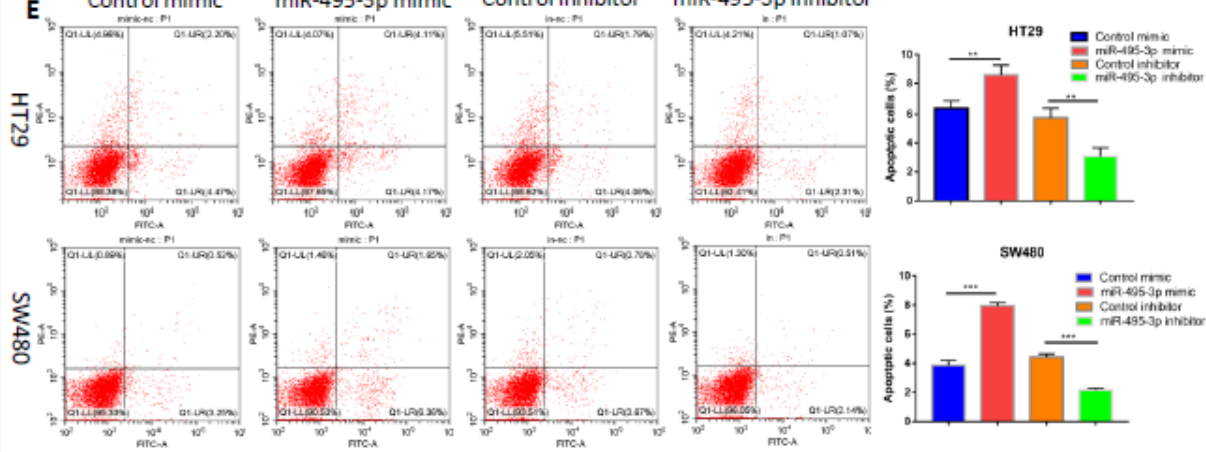

Figure 2 
miR-495-3p suppresses malignant phenotypes of CRC cells. (a) Relative CRC cell growth rates at $12 \mathrm{~h}, 24$ h, 36 h, 48 h, and $60 \mathrm{~h}$ after knockdown or upregulation of miR-495-3p were measured using a CCK-8 assay. (b) The effect of miR-495-3p on CRC cell proliferation was assessed by an EdU assay. (c) Relative EDU positive cell numbers after miR-495-3p knockdown and overexpression. (d) Transwell assays were performed to evaluate cellular migration after miR-495-3p knockdown and overexpression. (e) Flow cytometry apoptosis experiments were used to measure the apoptosis rate of HT-29 and SW480 cells after miR-495-3p knockdown and overexpression. * $p<0.05$, ** $p<0.01$, and $* * * p<0.001$

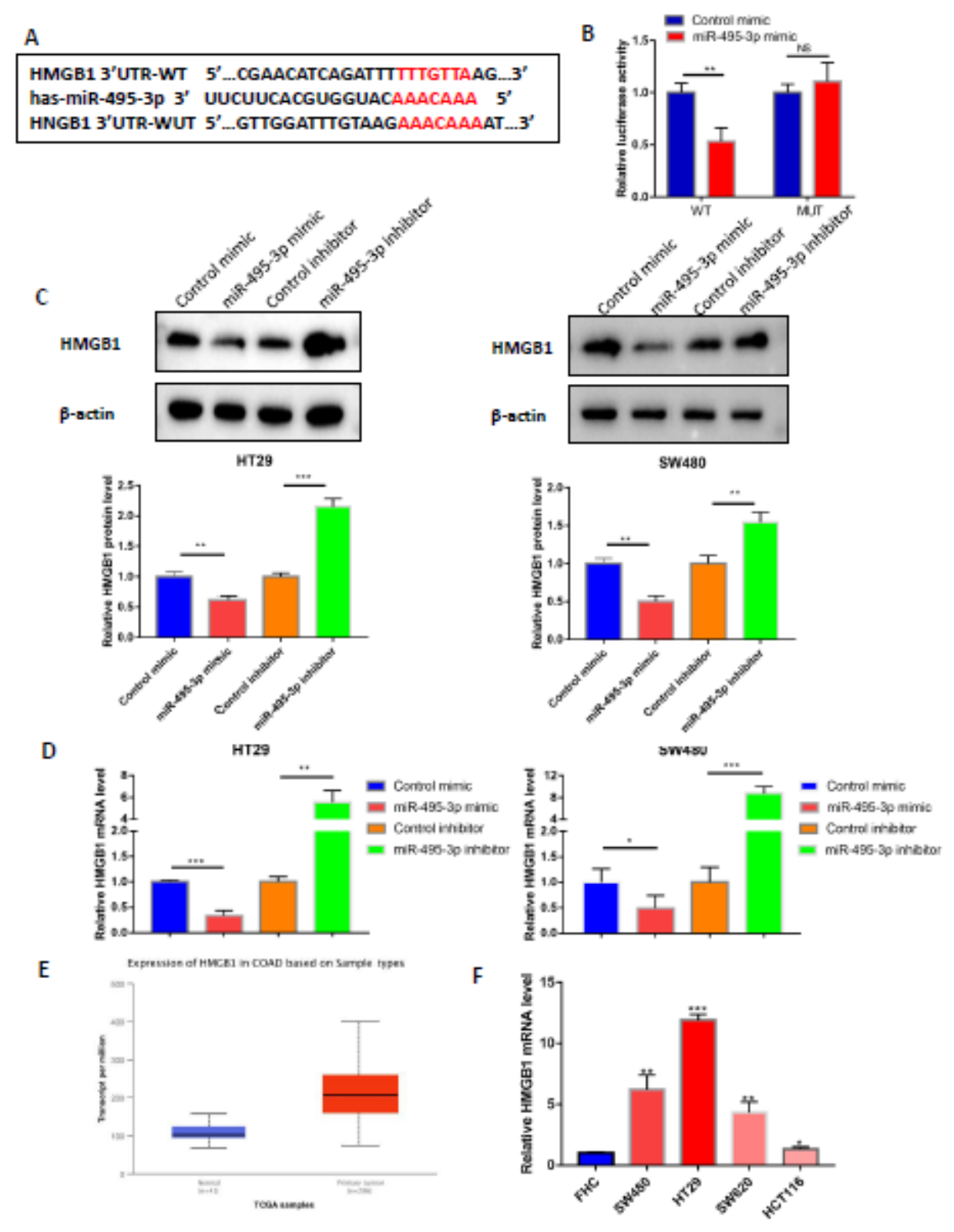

Figure 3

HMGB1 is overexpressed in CRC and is the target gene of miR-495-3p. (a) Target genes of miR-495-3p were predicted using TargetScan and RNAhybrid. $₫ \mathrm{~b} \otimes T$ he relative luciferase activity was measured by

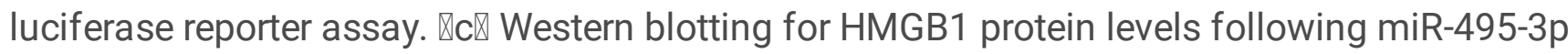
mimic/inhibitor transfection. (d) HMGB1 mRNA levels in HT29 and SW480 cells after miR-495-3p knockdown and overexpression assessed by RT-qPCR. (e) TCGA database analysis of HMGB1 expression 
in CRC. (f) HMGB1 mRNA levels in HT29, SW480, SW620, HCT116 and FHC cells assessed by RT-qPCR. * $p<0.05, * * p<0.01$, and $* \star * p<0.001$
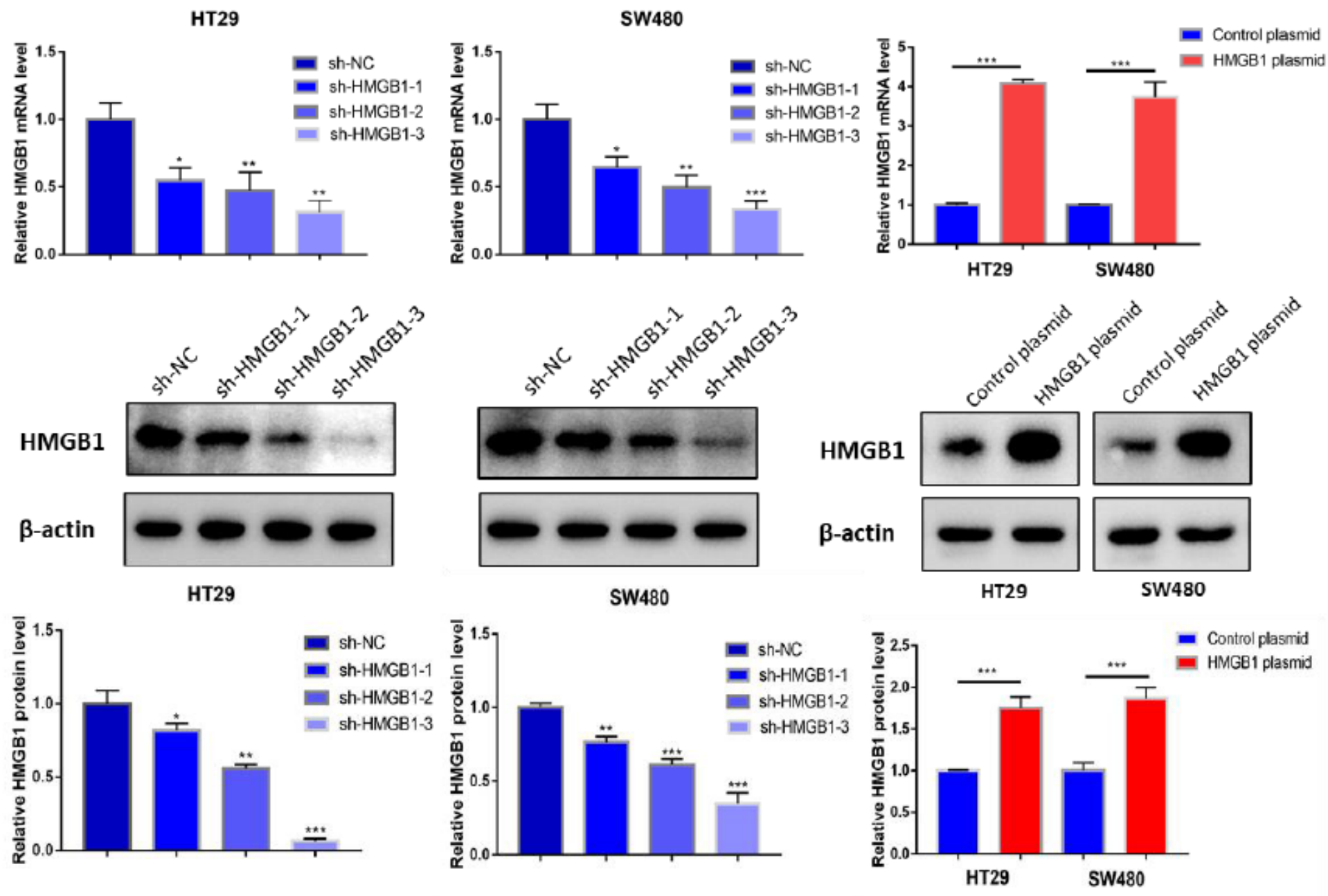

Figure 4

The efficiency of HMGB1 knocking-down and overexpressing was assessed by qPCR as well as Western blotting. ${ }^{*} p<0.05,{ }^{* \star} p<0.01$, and ${ }^{* \star *} p<0.001$ 

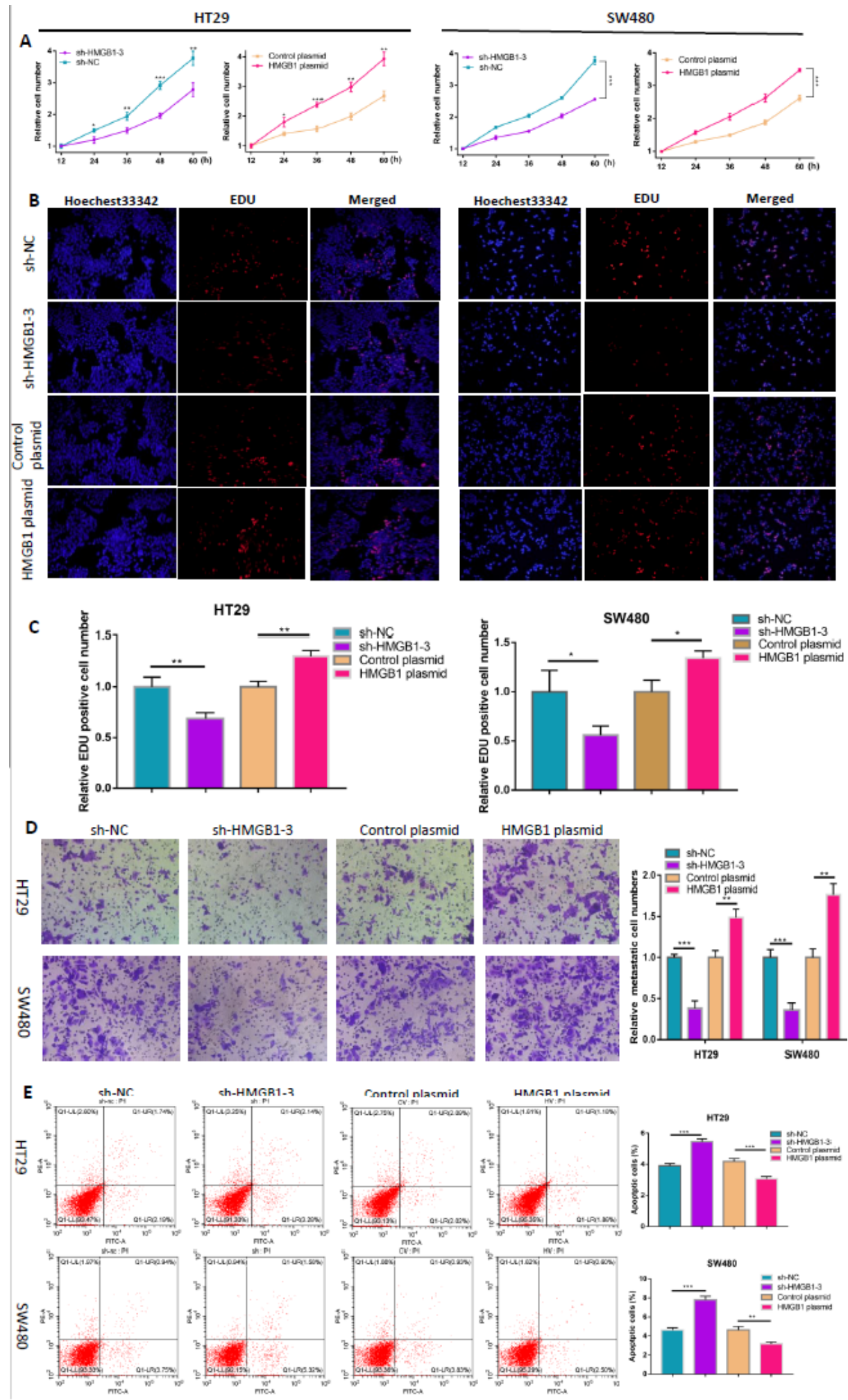

\section{Figure 5}

HMGB1 facilitates malignant phenotypes of CRC cells. (a) Relative CRC cell growth rates at $12 \mathrm{~h}, 24 \mathrm{~h}, 36$ h, $48 \mathrm{~h}$, and $60 \mathrm{~h}$ after knockdown or upregulation of HMGB1 were measured using a CCK-8 assay. (b) The effect of HMGB1 on CRC cell proliferation was assessed by an EdU assay. (c) Relative EDU positive cell numbers after HMGB1 knockdown and overexpression. (d) Transwell assays were performed to evaluate cellular migration after HMGB1 knockdown and overexpression. (e) Flow cytometry apoptosis 
experiments were used to measure the apoptosis rate of HT-29 and SW480 cells after HMGB1 knockdown and overexpression. ${ }^{*} p<0.05,{ }^{*} p<0.01$, and ${ }^{* *} p<0.001$

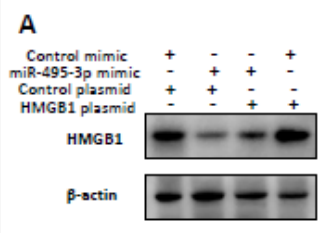

HT29
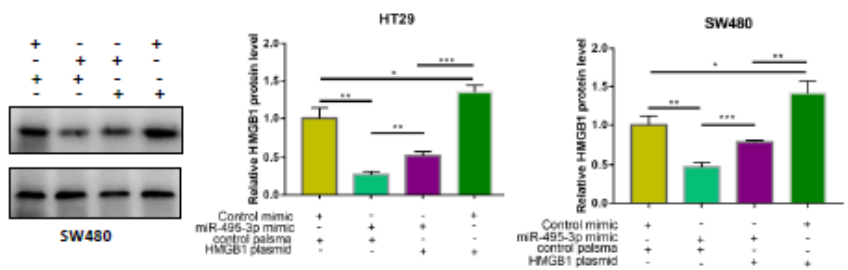

B

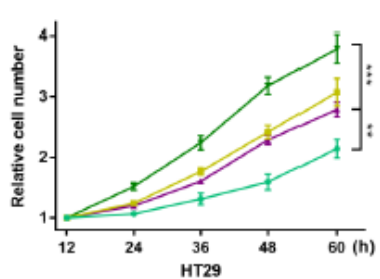

- $\begin{gathered}\text { HMGB1 plasmid } \\ + \text { +ontrol mimic }\end{gathered}-\begin{gathered}\text { mir-4yD-3p mimıc } \\ + \text { HMGB1 plasmid }\end{gathered}$ $\begin{array}{ll}+ \text { Control mimic } & \text { +HMGB1 plasmid } \\ \text { Control mimic } & \text { miR-495-3p mimic }\end{array}$

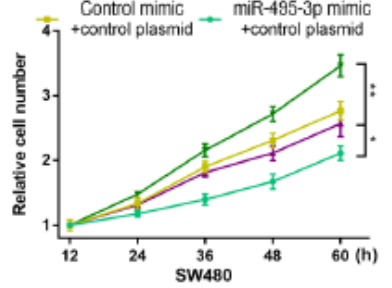

C control mimic +

miR-495-3p
mimic + contro

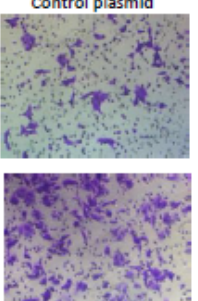

miR-495-3p

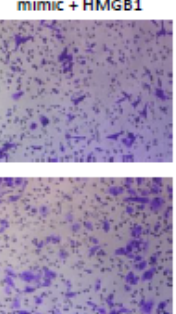

Control mimic +
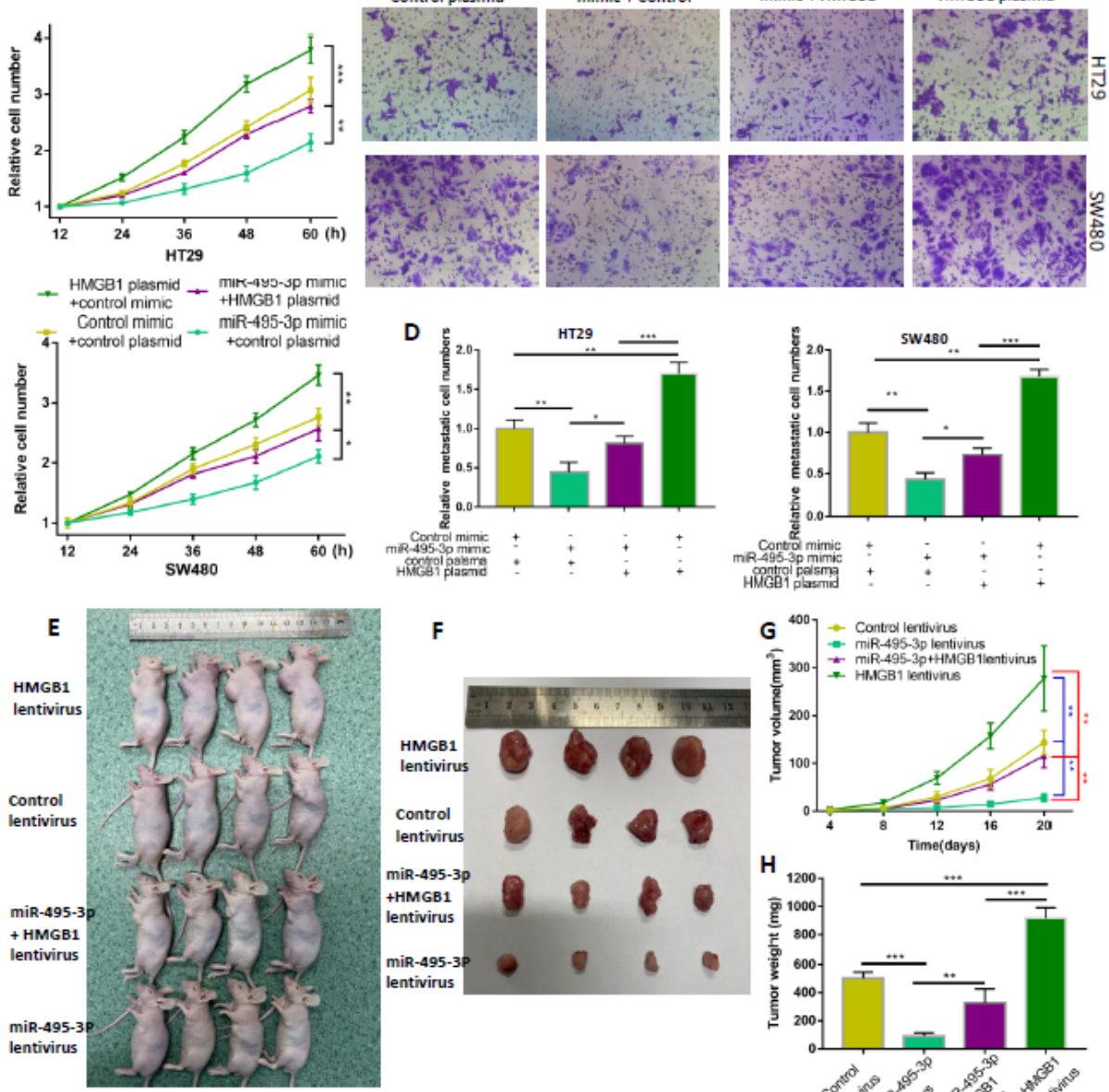

lentivirus

miR-495-3p +HMGB1 lentivirus miR-495-3P $9 \quad 9 \quad 6$ lentivirus

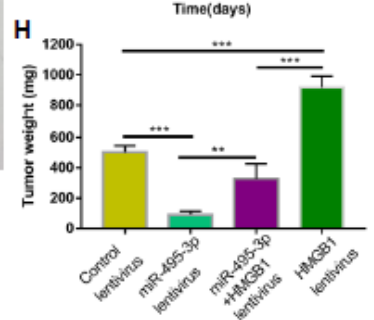

$$
\text { J }
$$
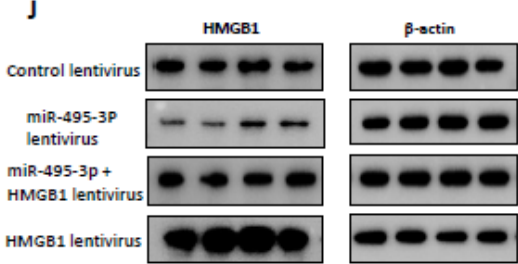

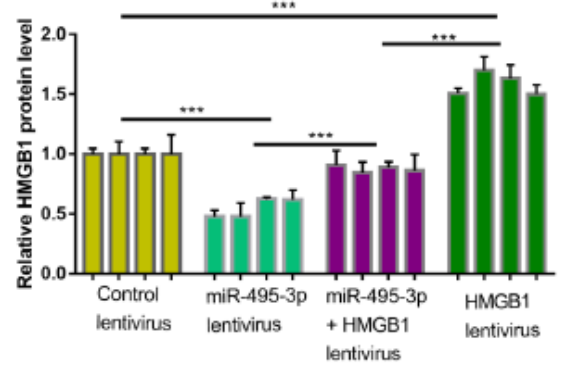

Figure 6

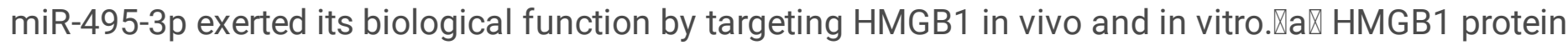
levels were restored after co-transfection with miR-495-3p mimics and HMGB1 plasmid in HT19 and SW480 cells. (b) Overexpression of HMGB1 attenuated the effect of miR-495-3p on the proliferation of 
CRC cells. (c, d) Overexpression of HMGB1 attenuated the effect of miR-495-3p on the migration of CRC cells. (e, f) Representative images of implanted mice and dissected tumor tissues. The HMGB1 lentivirus group with the largest tumor volume were placed at the front, and the miR-495-3p lentivirus group with the smallest tumor volume were placed at the end for the sake of facilitating the observation of tumor size. (g) Growth curves of the tumors. Tumor volumes were measured using a slide caliper every 4 days $(n=4)$. (h) Weights of xenograft tumor in different groups $(n=4)$. (j) HMGB1 protein levels in xenograft tumor tissues were detected by western blot analysis. ${ }^{*} p<0.05,{ }^{* *} p<0.01$, and ${ }^{* \star *} p<0.001$ 